\title{
Water in Sunspots and Stars
}

\author{
Peter F. Bernath \\ Department of Chemistry, University of Waterloo, 200 University \\ Avenue West, Waterloo, Ontario, Canada N2L 3G1
}

\begin{abstract}
Water is found in a wide variety of cool stars and substellar objects such as brown dwarfs. The detection of water vapor in such objects is reviewed.
\end{abstract}

\section{Introduction}

The main problem with the astronomical spectroscopy of water is the massive blanket of telluric water vapor. Atmospheric absorption has largely blocked the detection of cool water from the ground. Historically, two types of ground-based water observations are possible: masers and "steam bands". Maser transitions between highly-excited levels (e.g., $6_{16} \rightarrow 5_{23}$ at $22 \mathrm{GHz}$; Cheung et al. 1969) can be detected by radio techniques. Water masers are typically seen in masslosing, late-type stars and are often associated with star formation (e.g., Xiang \& Turner 1995). While kinematics can be readily extracted from maser data, the determination of abundances is extremely difficult.

\section{Mira variables}

Water is a light molecule with large rotational constants $\left(\mathrm{A}_{0}=28.9 \mathrm{~cm}^{-1}, \mathrm{~B}_{0}=\right.$ $14.5 \mathrm{~cm}^{-1}$ and $\mathrm{C}_{0}=9.3 \mathrm{~cm}^{-1}$ ) so that transitions between highly-excited levels are often shifted away from transitions between lower energy levels. This means that rotational and vibration-rotation transitions (water has no useful electronic transitions) associated with hot water can penetrate the earth's atmosphere. The overtone spectra of hot water vapour ("steam bands") can be detected in many cool stars. For example, Spinrad et al. (1966) found the stellar water band at $9278 \AA$ to be strong in late M-type stars, particularly Mira variables. The steam bands vary with the phase of the Miras and can be used to study stellar structure. For example, water is found (Hinkle \& Barnes 1979) in at least two layers around the star ("warm", $T \sim 1700 \mathrm{~K}$ found near the photosphere and "cool", $T \sim 1100 \mathrm{~K}$ in an overlying region).

\section{M-Giants and supergiants}

Water has also been identified in early $\mathrm{M}$ giants and supergiants such as $\alpha$ Ori, $\alpha$-Sco and $\mu$-Cep. The original identifications were based on the balloonborne Stratoscope II observations of $1.4 \mu \mathrm{m}$ and $1.9 \mu \mathrm{m}$ water bands in 1964 
(Tsuji 2000a). Water is relatively weak in these sources but can also be seen near $13 \mu \mathrm{m}$ through pure rotational transitions (Jennings \& Sada 1998) and in oxygen-rich circumstellar outflows (Neufeld et al. 1996, 1999; Justtanont et al. 1996). The water is not found in the photospheres of the early $M$ supergiants but in a circumstellar cloud ( $T \sim 1500 \mathrm{~K}$, cf. "cool" layer in Miras) dubbed the "MOLsphere" by Tsuji $(2000 \mathrm{~b})$. The pure rotational water lines in the far infrared spectra $(\sim 40 \mu \mathrm{m})$ of $\mu$-Cep recorded by the Infrared Space Observatory (ISO) satellite appear in emission.

The solution to the telluric water interference problem is to record spectra from satellites. The ISO satellite has found water in a large number of sources including star-forming regions such as Orion (Harwit et al. 1998; Wright et al. 2000), late M giants and Miras (Tsuji et al. 1997; Truong-Bach et al. 1999). Very recently, the first data from the Submillimeter Wave Astronomy Satellite (SWAS) has been published (Melnick et al. 2000). SWAS can detect water using the low-lying $1_{10}-1_{01}$ transition at $557 \mathrm{GHz}$. Because of the long wavelength and small diameter of the antenna, SWAS can detect water in extended objects such as Orion much more easily than compact sources such as stars.

\section{M-dwarfs and Brown Dwarfs}

Water is also present in the photosphere of M-dwarf stars. It starts to appear at $2.22 \mu \mathrm{m}$ in objects (Hinkle 2000) as early as M2 (e.g., G $\ell$ 411) and strengthens considerably by M6 (e.g., $G \ell$ 406). As the surface temperature of the dwarf drops below about $2000 \mathrm{~K}$, hydrogen burning in the core is no longer possible and the objects are called brown dwarfs. There are two types of brown dwarfs, $\mathrm{L}$ and $\mathrm{T}$, both of which show prominent water bands. In the temperature range of $\sim 1200-1800 \mathrm{~K}$, the $\mathrm{L}$ dwarfs have strong $\mathrm{FeH}$ and $\mathrm{CrH}$ bands but with weak TiO (Kirkpatrick et al. 1999). As the temperature drops even lower the metal hydrides disappear and the near infrared spectra of $\mathrm{T}$ dwarfs ("methane brown dwarfs") are dominated by hot water and hot methane (Leggett et al. 2000).

\section{Sunspots and Laboratory Experiments}

An important advance in the spectroscopy of hot water occurred as a result of the detection of water vapor in sunspots. At $5800 \mathrm{~K}$, the Sun's photosphere is too hot for water to exist. Temperatures as low as $\sim 3000 \mathrm{~K}$, however, are found in sunspots. We noticed a large number of unassigned lines in two sunspot atlases (Wallace et al. 1996). We suspected that these lines were due to hot water but the available laboratory data were inadequate to confirm this. We, therefore, recorded new laboratory emission spectra of hot water at $1800 \mathrm{~K}$ in the infrared region. Comparison of the laboratory emission spectra of $\mathrm{H}_{2} \mathrm{O}$ and the sunspot absorption spectra identified most of the unassigned sunspot lines, (Wallace et al. 1995) as $\mathrm{H}_{2} \mathrm{O}$ lines ("Water on the Sun").

Only a small fraction of the new water lines could be assigned quantum numbers. The water molecule is so light that the traditional power series expressions for the rotational energy levels are divergent. This massive breakdown of perturbation theory makes the assignment of quantum numbers to the lines very difficult. We began a collaboration with the theoreticians O. Polyansky 
and J. Tennyson to apply more sophisticated approaches to the assignment of our spectra. Recently we have made a breakthrough in the prediction of the spectra of hot water (Polyansky et al. 1997a).

The hot water lines in the laboratory and in the sunspot have been assigned by direct calculation of the infrared spectra starting with an ab initio potential energy surface. This surface was partially corrected for the breakdown of the Born-Oppenheimer approximation and used in a variational calculation of the energy levels. Although the predicted line positions were still in error, they were close enough to make the necessary assignments. So far we have recorded laboratory emission spectra in $400-6000 \mathrm{~cm}^{-1}$ region (Polyansky et al. 1997b; Zobov et al. 1999, 2000) and made assignments in the sunspot spectrum in the $\mathrm{N}$ band $(10-13 \mu \mathrm{m})$ as well as L band $(3-4 \mu \mathrm{m})$ and K-band $(2.0-2.3 \mu \mathrm{m})$. An important application of our hot water work is the calculation of molecular water opacities (Allard et al. 1997) for various cool objects.

\section{References}

Allard, F. et al. 1997, ARA\&A, 35, 137

Cheung, A.C. et al. 1969, Nature, 221, 626

Harwit, M. et al. 1998, ApJ, 497, L105

Hinkle, K.H. \& Barnes, T.G. 1979, ApJ, 227, 923

Hinkle, K.H. 2000, private communication

Jennings, D.E. \& Sada, P.V. 1998, Science, 279, 844

Justtanont, K. et al. 1996, A\&A, 315, L217

Kirkpatrick, J.D. et al. 1999, ApJ, 519, 802

Leggett, S.K. et al. 2000, ApJ, 536, L35

Melnick, G.J. et al. 2000, ApJ, 539, L77

Neufeld, D.A. et al. 1996, A\&A, 315, L237

Neufeld, D.A. et al. 1999, ApJ, 517, L147

Polyansky, O.L. et al.1997a, Science, 277, 346

Polyansky, O.L. et al. 1997b, J. Mol. Spectrosc., 186, 422

Spinrad, H., Pyper, D.M., Newburn, R.L., \& Younkin, R.L. 1966, ApJ, 143, 291

Truong-Bach et al. 1999, A\&A, 345, 925

Tsuji, T., Ohnaka, K., Aoki, W., \& Yamamura, I. 1997, A\&A, 320, L1

Tsuji, T. 2000a, ApJ, 538, 801

Tsuji, T. 2000b, ApJ, 540, L99

Wallace, L., Bernath, P.F., Livingston, W., Hinkle, K., Busler, J., Guo, B., \& Zhang, K. 1995, Science, 268, 1155

Wallace, L., Livingston, W., Hinkle, K., \& Bernath, P.F. 1996, ApJS, 106, 165

Wright et al. 2000, A\&A, 358, 689

Xiang, D. \& Turner, B.E. 1995, ApJS, 99, 121

Zobov N.F. et al. 2000, ApJ, 530, 994

Zobov N.F. et al. 1999, J. Mol. Spectrosc., 193, 118 